\title{
Microwave-assisted synthesis of curcumin analogs
}

\author{
Christie E. Nichols, Dani Youssef, ${ }^{\text {a }}$ Robert G. Harris, and Amitabh Jha* \\ Department of Chemistry, Acadia University, Wolfville, NS, Canada \\ ${ }^{a}$ Département des Sciences, Université Sainte-Anne, Church Point, NS, Canada \\ E-mail : amitabh.jha@,acadiau.ca
}

\begin{abstract}
Curcumin, a 1,7-diaryl-1,6-heptadiene-3,5-dinone, has attracted considerable attention worldwide owing to its outstanding biological properties. Microwave-assisted expeditious synthesis of numerous cyclic analogs of curcumin under solvent-free and environmentallybenign conditions in moderate to excellent yields is reported here.
\end{abstract}

Keywords: Curcumin, microwave, aldol condensation, solvent-free, diarylheptanoids

\section{Introduction}

Curcumin (1) is a phytochemical obtained from Curcuma longa, commonly known as turmeric, a spice widely used in South-East Asia. It has attracted a lot of attention due to its promising biological properties to treat cancer, ${ }^{1}$ Alzheimer's disease, ${ }^{2}$ HIV,${ }^{3,4}$ chronic inflammations, ${ }^{2}$ oxidative stress, ${ }^{5}$ and cystic fibrosis. ${ }^{6}$ Curcumin underwent clinical trial for cancer owing to its prominent activity as an antitumor and chemopreventive agent. ${ }^{7}$ However, this trial ceased due to poor bioavailability of the molecule. ${ }^{8,9}$ Clinical trials are ongoing to test the efficacy of curcumin against Alzheimer's disease ${ }^{10}$ and cystic fibrosis. ${ }^{11}$ Intense research is also being undertaken to modify the structure of curcumin so as to increase the bioavailability and potency while maintaining the relative non-toxic nature of this natural product. ${ }^{42-17}$<smiles>COc1cc(/C=C/C(=O)CC(=O)/C=C/c2ccc(O)c(OC)c2)ccc1O</smiles>

Figure 1. Tautomeric structure of curcumin (1). 
We are interested in the development of novel curcumin analogs with improved biological profiles. $^{16}$ Although curcumin is a simple symmetrical $\beta$-diketone, its synthesis is not a straightforward di-aldol condensation on 2,4-pentadione. ${ }^{18}$ The C-3 of 2,4-pentadione bears more acidic protons than those on $\mathrm{C}-1 / \mathrm{C}-5$ and therefore aldol condensations on terminal methyl groups ( $\mathrm{C}-1$ and $\mathrm{C}-5)$ must be carried out successively via the dienolate; this is hard to obtain and reaction at C-3 often leads to side products. ${ }^{19}$ Use of boron-based protection of the 1,3diketone circumvents the Knoevenagel condensation at C-3 and facilitates aldol condensations at $\mathrm{C}-1$ and $\mathrm{C}-5$ of 2,4-pentadione. ${ }^{18} \mathrm{~A}$ boron-based reagent such as boron oxide, boric acid and tributoxyboron complex as Lewis acids with the $\beta$-diketone systems and consequently reduces the nucleophilicity of the $\mathrm{C}-3$ position and the reaction occurs at the terminal active methylenes resulting in diarylheptanoids (Scheme 1). ${ }^{17,18,20}$

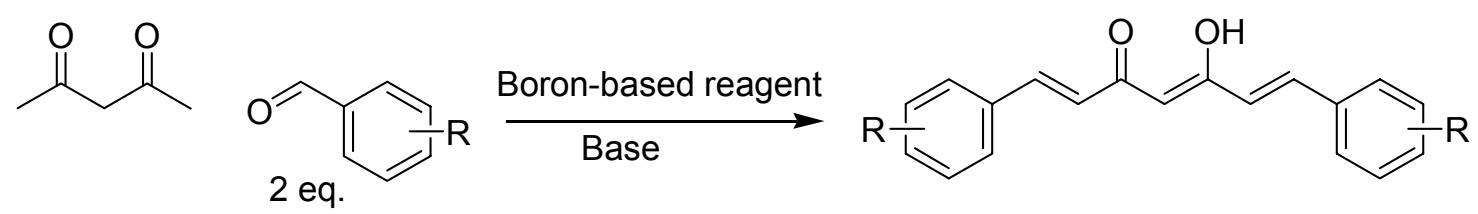

Scheme 1. Generic reaction for the preparation of diarylheptanoides like curcumin.

Unlike 2,4-pentadione, 2-acetylcycloalkanones not only have a more nucleophilic and more crowded active methine, but are also very prone to ring cleavage under aqueous basic conditions. ${ }^{21}$ A procedure reported for condensation of aromatic aldehydes on cyclic $\beta$-diketones involved the use of 1 molar equivalent of boron oxide in presence of catalytic amounts of morpholine and acetic acid under solvent-free heating conditions. ${ }^{20}$ The complexation with boron oxide also protects the 2 -acetylcycloalkanones from potential ring cleavage reaction. ${ }^{20}$ The reported yields under these conditions are moderate to good; however, the work-up conditions pose difficulties in purification of compounds.

We herein report a modification of this procedure with significant improvement in yields, reaction time and purity of isolated products.

\section{Results and Discussion}

Numerous microwave-assisted aldol condensations have been reported ${ }^{22-24}$ but the use of microwave energy in carrying out boron-assisted regioselective aldol condensation was not found in literature. Owing to the simplicity, rapidity, turnover and environment friendliness, use of microwave in organic synthesis has become very popular. ${ }^{22-25}$ Since the reported procedure for synthesis of carbocyclic analogs of curcumin involved heating conditions, it appeared logical to attempt their synthesis under microwave irradiation conditions. Moderate to excellent yields of the desired compounds were obtained when the reaction mixture was irradiated for 1 minute by 
microwaves. It should be noted that the reactions were carried out in a conventional microwave oven at highest power where the control of the reaction course is not ideal; however we found these reactions to be qualitatively (based on tlc intensities) reproducible.

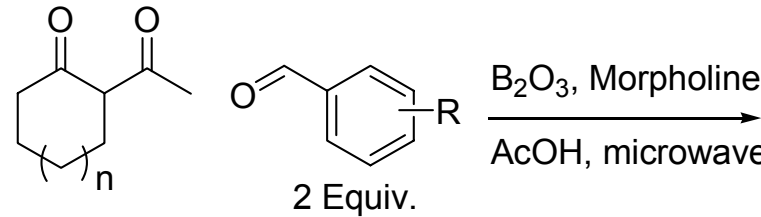

$\mathrm{n}=0,1$

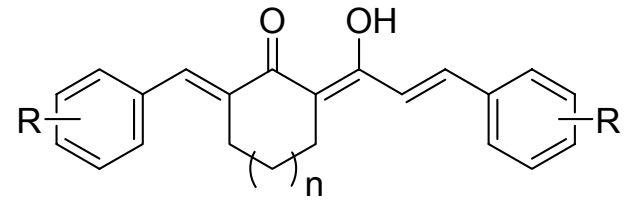

2a-h $n=0$ 3a-g $n=1$

Scheme 1. Synthetic scheme for making compounds $\mathbf{2 a - h}$ and 3a-g.

A modified work-up procedure made the isolation and purification of compounds simpler. As indicated in the experimental section, the yields of the compounds made under this investigation under microwave-assisted conditions are consistently higher than those reported under conventional conditions. Although the work-up conditions are generally product specific, the work-up of relatively non-polar compounds was straightforward. Digestion in methanol produced reasonably pure product in powder form. Polar products (2d, 2f, 3d and 3f) containing a hydroxyl group had appreciable solubility in methanol and were purified by column chromatography.

The compounds were characterized by spectroscopic means and by comparison to reported data wherever possible. ${ }^{20}$ NMR spectra revealed that the compounds exist in enol form in solution. The H-bonded hydroxyl proton of the enol form expected in highly desheilded region $(>\delta 13)$ was not always seen; the active methine (-COCHCO-) of the diketo tautomer was never observed. When subjected to HR-MS, all compounds concurred very well with calculated values. The biological activity of these compounds will be published subsequently.

\section{Conclusions}

In conclusion, an efficient, expeditious and simple procedure for the synthesis of carbocyclic curcumin analogues has been developed. It furnishes the desired compound with greater purity and moderate to excellent yields.

\section{Experimental Section}

General Procedures. All chemicals and reagents were obtained from Aldrich Chemical Co. Column chromatography purifications were undertaken using silica gel (230-400 mesh) obtained from Silicycle. ${ }^{1} \mathrm{H}$ NMR and ${ }^{13} \mathrm{C}$ NMR were recorded on Bruker AV300 NMR spectrometer. EI- 
MS and HR-MS spectra were obtained on CEC 21-110B Sector instrument. Melting points were recorded on an electro-thermal apparatus and are uncorrected. UV-Vis and IR spectra were recorded on LKB Biochrom Ultraspec Plus 4054 and Nicolet Avatar 330FT-IR spectrophotometers respectively. A domestic $1.2 \mathrm{cu}$. ft. microwave oven equipped with a turntable manufactured by Kenmore with $4 \mathrm{kV}$ DC operating voltage was used for the microwaveassisted reactions. The highest power $(900 \mathrm{~W})$ and operating frequency $(2450 \mathrm{MHz})$ were employed.

General synthesis of carbocyclic curcumin analogs 2 a-h and 3a-g. The cycloalkanone (10 mmol) was mixed with the boron oxide $(10 \mathrm{mmol})$ in a $50 \mathrm{~mL}$ Erlenmeyer flask. The appropriate aromatic aldehyde $(20 \mathrm{mmol})$, acetic acid $(50 \mathrm{mg})$, and morpholine $(50 \mathrm{mg})$ were then added. The reaction was irradiated with the microwave at high power for 1 minute. The flask was cooled for 2 minutes and then methanol $(30 \mathrm{~mL})$ was added. This mixture was then sonicated until a fine powder was obtained. The product so obtained was filtered and washed with cold methanol. Compounds 2d, 2f, 3d and 3f did not respond favourably to methanol treatment and consequently they were purified by silica gel column chromatography using dichloromethane-methanol as gradient solvent for elution.

2-[(E)-(3-Phenylacryloyl)]-5-[1-phenylmeth-(E)-ylidene]cyclopentanone (2a). Yellow solid; yield 83\% (lit. ${ }^{20}$ yield 75\%); m.p. $163-165{ }^{\circ} \mathrm{C}$ (lit. ${ }^{20}$ m.p. $\left.165-168{ }^{\circ} \mathrm{C}\right) .{ }^{1} \mathrm{H}$ NMR $(300 \mathrm{MHz}$, $\left.\mathrm{CDCl}_{3}\right): \delta 2.92\left(2 \mathrm{H}, \mathrm{t}, \mathrm{J}=6.0 \mathrm{~Hz}, \mathrm{CH}_{2}\right), 3.04-3.19\left(2 \mathrm{H}, \mathrm{m}, \mathrm{CH}_{2}\right), 6.71(1 \mathrm{H}, \mathrm{d}, \mathrm{J}=15.7 \mathrm{~Hz}, E-$ vinylic $\mathrm{H}), 7.34-7.67(11 \mathrm{H}, \mathrm{m}, \mathrm{ArH}$, vinylic- $\mathrm{H})$ and $7.73(1 \mathrm{H}, \mathrm{d}, \mathrm{J}=15.7 \mathrm{~Hz}, E$-vinylic $\mathrm{H}) .{ }^{13} \mathrm{C}$ NMR (75 MHz, $\left.\mathrm{CDCl}_{3}\right): \delta 23.45,26.99,114.36,120.69,128.44,129.13,129.23,129.34,130.40$, 130.66, 131.06, 135.76, 136.46, 139.37, 140.49, 171.06 and 193.76. IR, KBr Disc, v: 3418, 3053, 2953, 1635, 1442, 758 and $689 \mathrm{~cm}^{-1}$. UV-Vis MeOH, $\lambda_{\max }: 240,313$ and $374 \mathrm{~nm}$. MS (EI) 70eV, m/z (rel. intensity): $302\left(\mathrm{M}^{+}, 100\right), 225$ (4), 197 (3.7) and 131 (3.4). HRMS calculated for $\mathrm{C}_{21} \mathrm{H}_{18} \mathrm{O}_{2}$ : 302.1307, found: 302.1318 .

2-[(E)-3-(4-Chlorophenyl)acryloyl]-5-[1-(4-chlorophenyl)-meth-(E)-ylidene]-cyclopentanone (2b). Yellow solid; yield 77\%; m.p. 223-225 ${ }^{\circ} \mathrm{C} .{ }^{1} \mathrm{H}$ NMR (300 MHz, DMSO-d 6 ): $\delta 2.91$ $\left(2 \mathrm{H}, \mathrm{t}, \mathrm{J}=5.4 \mathrm{~Hz}, \mathrm{CH}_{2}\right), 3.00-3.04\left(2 \mathrm{H}, \mathrm{m}, \mathrm{CH}_{2}\right), 6.67(1 \mathrm{H}, \mathrm{d}, \mathrm{J}=15.6 \mathrm{~Hz}, E$-vinylic $\mathrm{H}), 7.30-7.54$ $\left(9 \mathrm{H}, \mathrm{m}, \mathrm{ArH}\right.$, vinylic-H) and $7.62(1 \mathrm{H}, \mathrm{d}, \mathrm{J}=15.6 \mathrm{~Hz}, E$-vinylic $\mathrm{H}) .{ }^{13} \mathrm{C} \mathrm{NMR}\left(75 \mathrm{MHz}, \mathrm{CDCl}_{3}\right) \delta$ (ppm): 23.42, 26.91, 114.54, 121.10, 129.40, 129.60, 129.62, 129.76, 131.74, 134.18, 134.87, 135.16, 136.32, 139.18, 139.76, 170.96 and 193.36. IR, KBr Disc, v: 3424, 2931, 1632, 1384, 1093, 819 and $518 \mathrm{~cm}^{-1}$. UV-Vis $\mathrm{MeOH}, \lambda_{\max }: 242 \mathrm{~nm}$. MS (EI) $70 \mathrm{eV}, m / z$ (rel. intensity): 372 ([M+2], 74), $370\left(\mathrm{M}^{+}, 100\right), 329$ (63), 248 (44.5), 191 (43) and 125 (30). HRMS calculated for $\mathrm{C}_{21} \mathrm{H}_{16} \mathrm{Cl}_{2} \mathrm{O}_{2}: 370.0527$, found: 370.0529 .

2-((E)-3-p-Tolylacryloyl)-5-[1-p-tolylmeth-(E)-ylidene]cyclopentanone (2c). Orange needles; yield 76\%; m.p. 202-204 ${ }^{\circ} \mathrm{C} .{ }^{1} \mathrm{H}$ NMR $\left(300 \mathrm{MHz} \mathrm{CDCl}_{3}\right): \delta 2.40\left(6 \mathrm{H}, \mathrm{s}, \mathrm{CH}_{3}\right), 2.89(2 \mathrm{H}, \mathrm{t}, \mathrm{J}=6.3$ $\left.\mathrm{Hz}, \mathrm{CH}_{2}\right), 3.00-3.09\left(2 \mathrm{H}, \mathrm{m}, \mathrm{CH}_{2}\right), 6.68(1 \mathrm{H}, \mathrm{d}, \mathrm{J}=15.3 \mathrm{~Hz}, E$-vinylic $\mathrm{H}), 7.21-7.26(4 \mathrm{H}, \mathrm{m}$, $\operatorname{ArH}), 7.36$ (1H, s, vinylic H), 7.46-7.50 (4H, m, ArH) and 7.63 (1H, d, J=15.3 Hz, E-vinylic H). ${ }^{13} \mathrm{C}$ NMR $\left(75 \mathrm{MHz}, \mathrm{CDCl}_{3}\right): \delta 21.91,23.43,26.99,114.13,119.66,128.43,128.76,129.89$, $130.08,130.69,131.02,133.08,133.74,138.45,139.55,140.36,140.82,170.95$ and 193.91. IR, 
KBr Disc, v: 3423, 2915, 1629, 1578, 1384 and $808 \mathrm{~cm}^{-1}$. UV-Vis MeOH, $\lambda_{\max }: 220$ and $305 \mathrm{~nm}$. MS (EI) 70eV, $m / z$ (rel. intensity): $330\left(\mathrm{M}^{+}, 100\right), 288$ (13), 171 (35), 145 (75), 115 (40) and 91 (26). HRMS calculated for $\mathrm{C}_{23} \mathrm{H}_{22} \mathrm{O}_{2}$ : 330.1620, found: 330.1627 .

2-[(E)-3-(4-Hydroxyphenyl)acryloyl]-5-[1-(4-hydroxyphenyl)meth-(E)-ylidene]-cyclopentanone (2d). Dark orange solid; yield 74\%; m.p. $>260{ }^{\circ} \mathrm{C} .{ }^{1} \mathrm{H}$ NMR (300 MHz, DMSO-d 6 ): $\delta 2.84$ $\left(2 \mathrm{H}, \mathrm{t}, \mathrm{J}=6.3 \mathrm{~Hz}, \mathrm{CH}_{2}\right), 2.91-2.94\left(2 \mathrm{H}, \mathrm{m}, \mathrm{CH}_{2}\right), 6.69(1 \mathrm{H}, \mathrm{d}, \mathrm{J}=15.6 \mathrm{~Hz}, E$-vinylic $\mathrm{H}), 6.81(2 \mathrm{H}$, $\mathrm{d}, \mathrm{J}=8.4, \mathrm{ArH}) 6.86(2 \mathrm{H}, \mathrm{d}, \mathrm{J}=8.4, \mathrm{ArH}), 7.16(1 \mathrm{H}, \mathrm{s}$, vinylic H), 7.45-7.51 (3H, m, ArH, $E-$ vinylic $\mathrm{H}), 7.60(2 \mathrm{H}, \mathrm{d}, \mathrm{J}=8.1 \mathrm{~Hz}, \mathrm{ArH}), 10.02(1 \mathrm{H}, \mathrm{brs}, \mathrm{OH})$ and $10.06(1 \mathrm{H}, \mathrm{brs}, \mathrm{OH}) .{ }^{13} \mathrm{C} \mathrm{NMR}$ $\left(75 \mathrm{MHz}, \mathrm{CDCl}_{3}\right): \delta 23.16,26.87,49.46,114.19,116.74,117.82,127.09,127.61,130.91$, 131.20, 133.13, 136.65, 140.41, 159.49, 160.56, 170.46 and 194.04. IR, KBr Disc, v: 3431, 1630, 1598, 1496, 1384, 1247, 1168, 1075 and $973 \mathrm{~cm}^{-1}$. UV-Vis MeOH, $\lambda_{\max }: 220$ and $251 \mathrm{~nm}$. MS (EI) 70eV, $m / z$ (rel. intensity): $334\left(\mathrm{M}^{+}, 4\right), 317$ (9), 254 (70) and 107 (94). HRMS calculated for $\mathrm{C}_{21} \mathrm{H}_{18} \mathrm{O}_{4}$ : 334.1205 , found: 334.1216. ESI-MS (-ve ion mode), $m / z$ (rel. intensity): 333 ([M-1], 100).

2-[(E)-3-(4-Methoxyphenyl)acryloyl]-5-[1-(4-methoxyphenyl)-meth-(E)-ylidene]-

cyclopentanone (2e). Dark yellow solid; yield 89\%; m.p. 200-201 ${ }^{\circ} \mathrm{C} .{ }^{1} \mathrm{H}$ NMR (300 MHz, $\left.\mathrm{CDCl}_{3}\right): \delta 2.89\left(2 \mathrm{H}, \mathrm{t}, \mathrm{J}=6.6 \mathrm{~Hz}, \mathrm{CH}_{2}\right), 2.96-3.05\left(2 \mathrm{H}, \mathrm{m}, \mathrm{CH}_{2}\right), 3.87\left(6 \mathrm{H}, \mathrm{s}, 2 \mathrm{xOCH}_{3}\right), 6.55(1 \mathrm{H}$, d, J=15.6 Hz, E-vinylic H) 6.91-6.99 (4H, m, ArH), 7.24-7.37 (2H, m, ArH) and 7.53-7.66 (4H, $\mathrm{m}, \mathrm{ArH}, 2 \mathrm{x}$ vinylic $\mathrm{H}) .{ }^{13} \mathrm{C} \mathrm{NMR}\left(75 \mathrm{MHz}, \mathrm{CDCl}_{3}\right): \delta 23.39,26.91,55.77,55.81,113.76$, $114.64,114.79,118.34,128.65,129.38,130.06,130.65,132.35,137.12,139.84,160.51,161.58$, 170.72 and 193.97. IR, KBr Disc, v: 3430, 2932, 1624, 1583, 1510, 1440, 1384, 1251, 1176, 1029 and $818 \mathrm{~cm}^{-1}$. UV-Vis $\mathrm{MeOH}, \lambda_{\max }$ : 220, 249 and $339 \mathrm{~nm}$. MS (EI) 70eV, m/z (rel. intensity): $362\left(\mathrm{M}^{+}, 100\right), 228$ (2.34), 202 (8), 159 (2.3) and 134 (3.2). HRMS calculated for $\mathrm{C}_{23} \mathrm{H}_{22} \mathrm{O}_{4}: 362.1518$, found: 362.1513 .

2-[(E)-3-(4-Hydroxy-3-methoxyphenyl)acryloyl]-5-[1-(4-hydroxy-3-methoxyphenyl)-meth(E)-ylidene]cyclopentanone (2f). Dark orange solid; yield 80\% (lit. ${ }^{20}$ yield 62\%); m.p. 221-224 ${ }^{\circ} \mathrm{C}$ (lit. ${ }^{20}$ m.p. $\left.224-227{ }^{\circ} \mathrm{C}\right) .{ }^{1} \mathrm{H}$ NMR $\left(300 \mathrm{MHz}, \mathrm{DMSO}-\mathrm{d}_{6}\right): \delta 2.87\left(2 \mathrm{H}, \mathrm{t}, \mathrm{J}=5.7 \mathrm{~Hz}, \mathrm{CH}_{2}\right), 2.96-$ $2.99\left(2 \mathrm{H}, \mathrm{m}, \mathrm{CH}_{2}\right), 3.83\left(3 \mathrm{H}, \mathrm{s}, \mathrm{OCH}_{3}\right), 3.84\left(3 \mathrm{H}, \mathrm{s}, \mathrm{OCH}_{3}\right), 6.73(1 \mathrm{H}, \mathrm{d}, \mathrm{J}=15.6 \mathrm{~Hz}$, E-vinylic H), 6.80-6.89 (3H, m, ArH), 7.10-7.19 (3H, m, ArH), 7.35 (1H, s, vinylic H), 7.49 (1H, d, J=15.6 $\mathrm{Hz}$, vinylic $\mathrm{H}), 9.63(1 \mathrm{H}$, brs, $\mathrm{OH})$ and $9.66(1 \mathrm{H}$, brs, $\mathrm{OH}) .{ }^{13} \mathrm{C} \mathrm{NMR}\left(75 \mathrm{MHz}, \mathrm{DMSO}-\mathrm{d}_{6}\right): \delta$ $23.29,26.79$, 56.45, 56.65, 112.42, 114.24, 115.06, 116.56, 116.72, 118.04, 123.91, 125.07, $127.57,128.10,131.29,136.86,140.83,148.53,148.84,149.02,150.13,170.50$ and 194.00. IR, KBr Disc, v: 3512, 3424, 2933, 1626, 1573, 1514, 1384, 1278, 1120, 1036 and $955 \mathrm{~cm}^{-1}$. UV-Vis $\mathrm{MeOH}, \lambda_{\max }: 220$ and $265 \mathrm{~nm}$. MS (EI) 70eV, $m / z$ (rel. intensity): $394\left(\mathrm{M}^{+}, 0.1\right), 261$ (95.3), 260 (100), 245 (40) and 137 (6). HRMS calculated for $\mathrm{C}_{23} \mathrm{H}_{22} \mathrm{O}_{6}: 394.1416$, found: 394.1410.

\section{2-[(E)-3-(3,4-Dimethoxyphenyl)acryloyl]-5-[1-(3,4-dimethoxyphenyl)-meth-(E)-ylidene]}

cyclopentanone (2g). Reddish brown soild; yield 90\%; m.p. 135-136 ${ }^{\circ} \mathrm{C} .{ }^{1} \mathrm{H} \mathrm{NMR}(300 \mathrm{MHz}$, $\left.\mathrm{CDCl}_{3}\right): \delta 2.90\left(2 \mathrm{H}, \mathrm{t}, \mathrm{J}=6.3 \mathrm{~Hz}, \mathrm{CH}_{2}\right), 3.00-3.10\left(2 \mathrm{H}, \mathrm{m}, \mathrm{CH}_{2}\right), 3.94(6 \mathrm{H}, \mathrm{s}, 2 \mathrm{xOCH}), 3.96(6 \mathrm{H}$, $\left.\mathrm{s}, 2 \mathrm{xOCH}_{3}\right), 6.54(1 \mathrm{H}, \mathrm{d}, \mathrm{J}=15.6 \mathrm{~Hz}$, vinylic $\mathrm{H}), 6.88-6.95(2 \mathrm{H}, \mathrm{m}, \mathrm{ArH}), 7.08-7.20(4 \mathrm{H}, \mathrm{m}$, $\mathrm{ArH}), 7.32(1 \mathrm{H}, \mathrm{s}$, vinylic $\mathrm{H})$ and $7.61(1 \mathrm{H}, \mathrm{d}, \mathrm{J}=15.6 \mathrm{~Hz}$, vinylic $\mathrm{H}) .{ }^{13} \mathrm{C} \mathrm{NMR}(75 \mathrm{MHz}$, 
$\left.\mathrm{CDCl}_{3}\right): \delta 23.45,26.88,56.29(\mathrm{x} 2), 56.38(\mathrm{x} 2), 110.29,111.54,112.70,113.43,113.80,118.48$, $122.78,124.32,128.87,129.63,130.99,137.32,140.22,149.28,149.61,150.23,151.34,170.65$ and 193.87. IR, KBr Disc, $v$ : 3431, 2927, 1620, 1592, 1513, 1384, 1255, 1137, 1019 and 613 $\mathrm{cm}^{-1}$. UV-Vis MeOH, $\lambda_{\text {max }}: 220$ and $264 \mathrm{~nm}$. MS (EI) 70eV, $m / z$ (rel. intensity): $422\left(\mathrm{M}^{+}, 0.2\right)$, 362 (4), 274 (100), 259 (21) and 231 (16). HRMS calculated for $\mathrm{C}_{25} \mathrm{H}_{26} \mathrm{O}_{6}$ : 422.1729, found: 422.1755. APCI-MS (-ve ion mode), $m / z$ (rel. intensity): 421 ([M-1], 100).

2-[(E)-3-(4-Nitrophenyl)acryloyl]-5-[1-(4-nitrophenyl)-meth-(E)-ylidene]-cyclopentanone (2h). Reddish brown solid; yield 84\%; m.p. 204-206 ${ }^{\circ} \mathrm{C} .{ }^{1} \mathrm{H}$ NMR $\left(300 \mathrm{MHz}, \mathrm{CDCl}_{3}\right): \delta 2.99$ $\left(2 \mathrm{H}, \mathrm{t}, \mathrm{J}=5.4 \mathrm{~Hz}, \mathrm{CH}_{2}\right), 3.09-3.13\left(2 \mathrm{H}, \mathrm{m}, \mathrm{CH}_{2}\right), 6.86(1 \mathrm{H}, \mathrm{d}, \mathrm{J}=15.6 \mathrm{~Hz}, E$-vinylic $\mathrm{H}), 7.62-7.83$ $(7 \mathrm{H}, \mathrm{m}, \mathrm{ArH}$, vinylic $\mathrm{H})$ and 8.28-8.35 (3H, m, ArH, vinylic $\mathrm{H}) .{ }^{13} \mathrm{C} \mathrm{NMR}\left(75 \mathrm{MHz}, \mathrm{CDCl}_{3}\right): \delta$ 23.57, 26.91, 115.77, 124.41, 124.27, 129.00, 129.85, 131.52, 132.29, 138.25, 140.57, 142.05, 143.16, 147.60, 148.13, 171.44 and 192.17. IR, KBr Disc, v: 3423, 1631, 1595, 1384, 1342, 1184, 855 and $756 \mathrm{~cm}^{-1}$. UV-Vis MeOH, $\lambda_{\max }$ : 220 and $355 \mathrm{~nm}$. MS (EI) 70eV, m/z (rel. intensity): $392\left(\mathrm{M}^{+}, 0.2\right), 259$ (100), 217 (5), 141 (1.5) and 74 (49). HRMS calculated for $\mathrm{C}_{21} \mathrm{H}_{16} \mathrm{~N}_{2} \mathrm{O}_{6}$ : 392.1008, found: 392.1012.

2-[(E)-(3-Phenylacryloyl)]-6-[1-phenylmeth-(E)-ylidene]cyclohexanone (3a). Yellow solid; yield $77 \%$ (lit. ${ }^{20}$ yield $72 \%$ ); m.p. $110-112{ }^{\circ} \mathrm{C}$ (lit. ${ }^{20}$ m.p. $\left.130-132{ }^{\circ} \mathrm{C}\right) .{ }^{1} \mathrm{H}$ NMR (300 MHz, $\left.\mathrm{CDCl}_{3}\right): \delta$ 1.77-1.86 $\left(2 \mathrm{H}, \mathrm{m}, \mathrm{CH}_{2}\right), 2.71\left(2 \mathrm{H}, \mathrm{t}, \mathrm{J}=6.0 \mathrm{~Hz}, \mathrm{CH}_{2}\right), 2.77-2.81\left(2 \mathrm{H}, \mathrm{m}, \mathrm{CH}_{2}\right), 7.15$ $(1 \mathrm{H}, \mathrm{d}, \mathrm{J}=15.6 \mathrm{~Hz}, E$-vinylic H), 7.33-7.44 (8H, m, ArH), 7.61-7.63 (2H, m, ArH), 7.75 (1H, s, vinylic $\mathrm{H})$ and $7.79(1 \mathrm{H}, \mathrm{d}, \mathrm{J}=15.6 \mathrm{~Hz}, E$-vinylic $\mathrm{H}) .{ }^{13} \mathrm{C} \mathrm{NMR}\left(75 \mathrm{MHz}, \mathrm{CDCl}_{3}\right): \delta 23.34,24.83$, $27.48,109.30,120.92$, 128.51, 128.71, 129.33, 130.27, 130.46, 130.57, 132.54, 133.79, 135.72, 136.76, 142.52, 179.02 and 185.88. IR, KBr Disc, v: 3447, 2947, 1631, 1610, 1674, 1443, 1339, 1276, 974, 926, 770 and $690 \mathrm{~cm}^{-1}$. UV-Vis, MeOH, $\lambda_{\max }: 237,320$ and $378 \mathrm{~nm}$. MS (EI), 70eV, $\mathrm{m} / \mathrm{z}$ (rel. intensity): $316\left(\mathrm{M}^{+}, 5.6\right), 228$ (100), 213 (8), 137 (1.5) and 115 (5.4). HRMS calculated for $\mathrm{C}_{22} \mathrm{H}_{20} \mathrm{O}_{2}: 316.1463$, found: 316.1482 .

2-[(E)-3-(4-Chlorophenyl)acryloyl]-6-[1-(4-chlorophenyl)meth-(E)-ylidene]-cyclohexanone (3b). Dark yellow solid; yield $73 \%$ (lit. ${ }^{20}$ yield $68 \%$ ); m.p. $173-175{ }^{\circ} \mathrm{C}$ (lit. ${ }^{20}$ m.p. $173-175{ }^{\circ} \mathrm{C}$ ). ${ }^{1} \mathrm{H}$ NMR (300 MHz, $\left.\mathrm{CDCl}_{3}\right): \delta 1.82\left(2 \mathrm{H}, \mathrm{m}, \mathrm{CH}_{2}\right), 2.68-2.74\left(4 \mathrm{H}, \mathrm{m}, 2 \mathrm{xCH}_{2}\right), 7.10(1 \mathrm{H}, \mathrm{d}$, $\mathrm{J}=15.3 \mathrm{~Hz}, E$-vinylic H), $7.34(6 \mathrm{H}, \mathrm{m}, \mathrm{ArH}), 7.52-7.59(2 \mathrm{H}, \mathrm{m}, \mathrm{ArH})$ and 7.68-7.77 (2H, m, vinylic $\mathrm{H}) .{ }^{13} \mathrm{C} \mathrm{NMR}\left(75 \mathrm{MHz}, \mathrm{CDCl}_{3}\right): \delta 23.22,24.75,27.45,109.45,121.31,128.98,129.61$, 129.83, 131.67, 132.55, 134.04, 134.15, 134.44, 135.13, 136.48, 141.17, 178.79 and 185.59. IR, KBr Disc, v: 2916, 2849, 1614, 1488, 1085, 1010, 973, 816 and $718 \mathrm{~cm}^{-1}$. UV-Vis MeOH, $\lambda_{\max }$ : 236, 316 and $384 \mathrm{~nm}$. MS (EI) $70 \mathrm{eV}, \mathrm{m} / z$ (rel. intensity): $384\left(\mathrm{M}^{+}, 100\right), 344$ (4), 217 (11), 194 (14) and 138 (12). HRMS calculated for $\mathrm{C}_{22} \mathrm{H}_{18} \mathrm{Cl}_{2} \mathrm{O}_{2}$ : 384.0684 , found: 384.0681.

2-((E)-3-p-Tolylacryloyl)-6-[1-p-tolylmeth-(E)-ylidene]cyclohexanone (3c). Orange needles; yield 71\%; m.p. $180-181{ }^{\circ} \mathrm{C} .{ }^{1} \mathrm{H}$ NMR $\left(300 \mathrm{MHz}, \mathrm{CDCl}_{3}\right): \delta 1.79-1.83\left(2 \mathrm{H}, \mathrm{m}, \mathrm{CH}_{2}\right), 2.40(6 \mathrm{H}$, s, $\left.2 \mathrm{xCH}_{3}\right), 2.69\left(2 \mathrm{H}, \mathrm{t}, \mathrm{J}=6.0 \mathrm{~Hz}, \mathrm{CH}_{2}\right), 2.75-2.80\left(2 \mathrm{H}, \mathrm{m}, \mathrm{CH}_{2}\right), 7.09(1 \mathrm{H}, \mathrm{d}, \mathrm{J}=15.6 \mathrm{~Hz}, E-$ vinylic H), 7.24-7.37 (4H, m, ArH), $7.35(2 \mathrm{H}, \mathrm{d}, \mathrm{J}=7.8 \mathrm{~Hz}, \mathrm{ArH}), 7.51(2 \mathrm{H}, \mathrm{d}, \mathrm{J}=7.8 \mathrm{~Hz}, \operatorname{ArH})$, $7.71(1 \mathrm{H}, \mathrm{s}$, vinylic $\mathrm{H}), 7.75(1 \mathrm{H}, \mathrm{d}, \mathrm{J}=15.6 \mathrm{~Hz}, E$-vinylic $\mathrm{H})$ and $17.20(1 \mathrm{H}$, brs, chelated $\mathrm{OH})$. ${ }^{13} \mathrm{C} \mathrm{NMR}\left(75 \mathrm{MHz}, \mathrm{CDCl}_{3}\right): \delta 21.78,21.93,23.35,24.82,27.58,109.11,119.91,128.70,129.47$, 
130.06, 130.53, 132.88, 133.02, 133.74, 133.95, 138.67, 141.02, 142.44, 179.11 and 185.83. IR, KBr Disc, v: 3022, 2936, 2857, 1613, 1510, 1174, 977 and $807 \mathrm{~cm}^{-1}$. UV-Vis MeOH, $\lambda_{\max }: 220$, 235 and $352 \mathrm{~nm}$. MS (EI) 70eV, m/z (rel. intensity): $344\left(\mathrm{M}^{+}, 100\right), 252$ (3.6), 197 (3.4) and 171 (2.7). HRMS calculated for $\mathrm{C}_{24} \mathrm{H}_{24} \mathrm{O}_{2}: 344.1776$, found: 344.1771 .

2-[(E)-3-(4-Hydroxyphenyl)acryloyl]-6-[1-(4-hydroxyphenyl)meth-(E)-ylidene]cyclohexanone (3d). Dark orange solid; yield 88\%; m.p. 214-216 ${ }^{\circ} \mathrm{C} .{ }^{1} \mathrm{H}$ NMR $(300 \mathrm{MHz}$, DMSO-d $\left.{ }_{6}\right): \delta 1.65-175\left(2 \mathrm{H}, \mathrm{m}, \mathrm{CH}_{2}\right), 2.62-2.70\left(4 \mathrm{H}, \mathrm{m}, 2 \mathrm{xCH}_{2}\right), 6.82-6.84(4 \mathrm{H}, \mathrm{m}, \mathrm{ArH}), 7.10$ $(1 \mathrm{H}, \mathrm{d}, \mathrm{J}=15.3 \mathrm{~Hz}, E$-vinylic H), 7.37 (2H, d, J=8.4 Hz, ArH), 7.51 (1H, s, vinylic H), 7.59-7.65 $(3 \mathrm{H}, \mathrm{m}, \mathrm{ArH}$, vinylic $\mathrm{H}), 9.92(1 \mathrm{H}, \mathrm{bs}, \mathrm{OH})$ and $10.06(1 \mathrm{H}, \mathrm{bs}, \mathrm{OH}) .{ }^{13} \mathrm{C} \mathrm{NMR}(75 \mathrm{MHz}$, $\left.\mathrm{CDCl}_{3}\right): \delta 23.41,24.29,27.65,109.15,116.34,116.72,117.93,126.92,127.54,130.92,131.60$, 132.98, 133.77, 142.91, 158.90, 160.79, 179.34 and 185.49. IR, KBr Disc, v: 3346, 2927m 1627, 1596, 1513, 1440, 1309, 1164 and $826 \mathrm{~cm}^{-1}$. UV-Vis, MeOH, $\lambda_{\max }: 220$ and $249 \mathrm{~nm}$. MS (EI) $70 \mathrm{eV}, m / z$ (rel. intensity): $348\left(\mathrm{M}^{+}, 0.9\right), 260$ (11.2), 244 (100), 147 (17.5) and 120 (72). HRMS calculated for $\mathrm{C}_{22} \mathrm{H}_{20} \mathrm{O}_{4}$ : 348.1361, found: 348.1341 .

\section{2-[(E)-3-(4-Methoxyphenyl)acryloyl]-6-[1-(4-methoxyphenyl)meth-(E)-ylidene]-}

cyclohexanone (3e). Dark orange solid; yield 91\% (lit. ${ }^{20}$ yield 8\%); m.p. $143-145{ }^{\circ} \mathrm{C}$ (lit. ${ }^{20}$ m.p. 146-148 $\left.{ }^{\circ} \mathrm{C}\right) .{ }^{1} \mathrm{H}$ NMR $\left(300 \mathrm{MHz}, \mathrm{CDCl}_{3}\right): \delta 1.80-1.84\left(2 \mathrm{H}, \mathrm{m}, \mathrm{CH}_{2}\right), 2.69(2 \mathrm{H}, \mathrm{t}, \mathrm{J}=6.0 \mathrm{~Hz}$, $\left.\mathrm{CH}_{2}\right), 2.76-2.79\left(2 \mathrm{H}, \mathrm{m}, \mathrm{CH}_{2}\right), 3.86\left(3 \mathrm{H}, \mathrm{s}, \mathrm{OCH}_{3}\right), 3.87\left(3 \mathrm{H}, \mathrm{s}, \mathrm{OCH}_{3}\right), 6.93-7.04(5 \mathrm{H}, \mathrm{m}, \mathrm{ArH}$, $E$-vinylic H), 7.42 (2H, d, J=8.7 Hz, ArH), 7.57 (2H, d, J=8.7 Hz, ArH), 7.69 (1H, s, vinylic H) and $7.75(1 \mathrm{H}, \mathrm{d}, \mathrm{J}=15.6 \mathrm{~Hz}, E$-vinylic $\mathrm{H}) .{ }^{13} \mathrm{C} \mathrm{NMR}\left(75 \mathrm{MHz}, \mathrm{CDCl}_{3}\right): \delta 23.28,24.79,27.63$, 55.74, 55.82, 108.85, 114.22, 114.77, 118.57, 128.54, 129.46, 130.39, 131.75, 132.20, 133.37 , 142.06, 159.94, 161.73, 179.13 and 185.68. IR, KBr Disc, v: 3432, 2947, 1630, 1597, 1384, 1253, 1169, 1025 and $829 \mathrm{~cm}^{-1}$. UV-Vis, MeOH, $\lambda_{\max }: 222$ and $271 \mathrm{~nm}$. MS (EI) 70eV, $m / z$ (rel. intensity): $376\left(\mathrm{M}^{+}, 8.2\right), 258$ (2.7), 178 (9), 135 (100). HRMS calculated for $\mathrm{C}_{24} \mathrm{H}_{24} \mathrm{O}_{4}$ : 376.1674, found: 376.1673 .

\section{2-[(E)-3-(4-Hydroxy-3-methoxyphenyl)acryloyl]-6-[1-(4-hydroxy-3-methoxyphenyl)-meth-} (E)-ylidene]cyclohexanone (3f). Dark orange solid; yield 86\% (lit. ${ }^{20}$ yield $75 \%$ ); m.p. 175-176 ${ }^{\circ} \mathrm{C}$ (lit. ${ }^{20}$ m.p. $\left.176-177{ }^{\circ} \mathrm{C}\right) .{ }^{1} \mathrm{H}$ NMR $\left(300 \mathrm{MHz}, \mathrm{CDCl}_{3}\right): \delta 1.81-1.85\left(2 \mathrm{H}, \mathrm{m}, \mathrm{CH}_{2}\right), 2.69(2 \mathrm{H}, \mathrm{t}$, $\left.\mathrm{J}=6.0 \mathrm{~Hz}, \mathrm{CH}_{2}\right), 2.79\left(2 \mathrm{H}, \mathrm{m}, \mathrm{CH}_{2}\right), 3.93\left(3 \mathrm{H}, \mathrm{s}, \mathrm{OCH}_{3}\right), 3.97\left(3 \mathrm{H}, \mathrm{s}, \mathrm{OCH}_{3}\right), 5.79(1 \mathrm{H}, \mathrm{brs}, \mathrm{OH})$, 5.89 (1H, brs, OH), $6.99-7.08$ (6H, m, ArH, E-vinylic H), 7.20 (1H, d, J=8.1 Hz, ArH), 7.67 $(1 \mathrm{H}, \mathrm{s}$, vinylic $\mathrm{H})$ and $7.75(1 \mathrm{H}, \mathrm{d}, \mathrm{J}=15.6 \mathrm{~Hz}, E$-vinylic $\mathrm{H}) .{ }^{13} \mathrm{C} \mathrm{NMR}\left(75 \mathrm{MHz}, \mathrm{CDCl}_{3}\right): \delta$ 23.45, 24.33, 27.71, 56.48, 56.66, 109.24, 112.46, 115.30, 116.36, 116.50, 118.13, 124.58, $124.78,127.40,128.01,131.14,134.12,143.33,148.26,148.37,148.85,150.40,179.43$ and 185.39. IR, KBr Disc, v: 3423, 3248, 2930, 1598, 1550, 1507, 1432, 1384, 1273, 1209, 1159 , 969 and $809 \mathrm{~cm}^{-1}$. UV-Vis, MeOH, $\lambda_{\max }: 220$ and $265 \mathrm{~nm}$. MS (EI) 70eV, $m / z$ (rel. intensity): $408\left(\mathrm{M}^{+}, 0.25\right), 232$ (46), 150 (100), 135 (2.3) and 107 (3). HRMS calculated for $\mathrm{C}_{24} \mathrm{H}_{24} \mathrm{O}_{6}$ : 408.1573, found: 408.1566 .

2-[(E)-3-(3,4-Dimethoxyphenyl)acryloyl]-6-[1-(3,4-dimethoxyphenyl)meth-(E)ylidene]cyclohexanone (3g). Dark orange needles; yield 92\%; m.p. 146-147 ${ }^{\circ} \mathrm{C} .{ }^{1} \mathrm{H}$ NMR (300 $\left.\mathrm{MHz}_{\mathrm{CDCl}}\right): \delta$ 1.84-1.86 (2H, m, $\left.\mathrm{CH}_{2}\right), 2.72\left(2 \mathrm{H}, \mathrm{t}, \mathrm{J}=5.7 \mathrm{~Hz}, \mathrm{CH}_{2}\right), 2.82\left(2 \mathrm{H}, \mathrm{m}, \mathrm{CH}_{2}\right), 3.92$ 
$\left(3 \mathrm{H}, \mathrm{s}, \mathrm{OCH}_{3}\right), 3.94\left(3 \mathrm{H}, \mathrm{s}, \mathrm{OCH}_{3}\right), 3.95\left(3 \mathrm{H}, \mathrm{s}, \mathrm{OCH}_{3}\right), 3.96\left(3 \mathrm{H}, \mathrm{s}, \mathrm{OCH}_{3}\right), 6.89-7.27(7 \mathrm{H}, \mathrm{m}$, ArH, E-vinylic H), 7.69 (1H, s, vinylic $\mathrm{H})$ and $7.75(1 \mathrm{H}, \mathrm{d}, \mathrm{J}=15.3 \mathrm{~Hz}, E$-vinylic $\mathrm{H}) .{ }^{13} \mathrm{C}$ NMR $\left(75 \mathrm{MHz}, \mathrm{CDCl}_{3}\right): \delta 23.39,24.80,27.68,56.31,56.33,56.37,56.40,108.89,110.53,111.27$, $111.55,113.78,118.71,123.16,123.81,128.80,129.73,132.00,133.64,142.41,149.02,149.62$ (x2), 151.52, 179.12 and 185.50. IR, KBr Disc, v: 3439, 2933, 2839, 1631, 1594, 1516, 1384, 1261, 1138, 1022 and $963 \mathrm{~cm}^{-1}$. UV-Vis MeOH, $\lambda_{\max }: 220$ and $263 \mathrm{~nm}$. MS (EI) $\mathrm{m} / z$ (rel. intensity): $436\left(\mathrm{M}^{+}, 29\right), 407$ (18), 246 (100), 208 (80) and 151 (16). HRMS calculated for $\mathrm{C}_{26} \mathrm{H}_{28} \mathrm{O}_{6}$ : 436.1886, found: 436.1879 .

\section{Acknowledgements}

Financial support from Nova Scotia Health Research Foundation (AJ, DY) and Natural Science and Engineering Research Council of Canada (AJ) is gratefully acknowledged. RGH thanks Cancer Care Nova Scotia for Norah Stephan Oncology Scholarship.

\section{References}

1. Sharma, R. A.; Gescher, A. J.; Steward, W. P. Eur. J. Cancer 2005, 41, 1955.

2. Lim, G. P.; Chu, T.; Yang, F.; Beech, W.; Frautschy, S. A.; Cole, G. M. J. Neurosci. 2001, 21,8370 .

3. De Clercq, E. Med. Res. Rev. 2000, 20, 323.

4. Mazumder, A.; Neamati, N.; Sunder, S.; Schulz, J.; Pertz, H.; Eich, E.; Pommier, Y. J. Med. Chem. 1997, 40, 3057.

5. Kopani, M.; Celec, P.; Danisovic, L.; Michalka, P.; Biro, C. Clin. Chim. Acta 2006, 364, 61.

6. Zeitlin, P. New Engl. J. Med. 2004, 351, 606.

7. National Cancer Institute, Clinical Development Plan: Curcumin. J. Cell. Biochem. 1996, $26 S, 72$.

8. Shoba, G.; Joy, D.; Joseph, T.; Majeed, M.; Rajendran, R.; Srinivas, P. S. Planta Med. 1998, $64,353$.

9. Sharma, R. A.; McLelland, H. R.; Hill, K. A.; Ireson, C. R.; Euden, S. A.; Manson, M. M.; Pirmohamed, M.; Marnett, L. J.; Gescher, A. J.; Steward, W. P. Clin. Cancer Res. 2001, 7, 1894.

10. National Institute of Aging, A phase II, double-blind, placebo-controlled study of the safety and tolerability of two doses of curcumin $\mathrm{C} 3$ complex versus placebo in patients with mild to moderate Alzheimer's disease, 2006, http://clinicaltrials.gov/show/NCT00099710.

11. Ramsey, B. A phase I safety and dose finding study of orally administered curcuminoids in adult subjects with cystic fibrosis who are homozygous for delta F508 cystic fibrosis 
transmembrane conductance regulator ( $\triangle \mathrm{F} 508 \quad$ CFTR $)$ mutation, 2005, http://www.clinicaltrials.gov/ct/show/NCT00219882.

12. Lee, S. L.; Huang, W. J.; Lin, W. W.; Lee, S. S.; Chen, C. H. Bioorg. Med. Chem. 2005, 13, 6175.

13. Ishida, J.; Ohtsu, H.; Tachibana, Y.; Nakanishi, Y.; Bastow, K. F. Nagai, M.; Wang, H. K.; Itokawa, H.; Lee, K. H. Bioorg. Med. Chem. 2002, 10, 3481.

14. Youssef, K. M.; El-Sherbeny, M. A.; El-Shafie, F. S.; Farag, H. A.; Al-Deeb, O.A.; Awadalla, S. A. A. Arch. Pharm. Pharm. Med. Chem. 2004, 337, 42.

15. Ohtsu, H.; Xiao, Z.; Ishida, J.; Nagai, M.; Wang, H.K.; Itokawa, H.; Su, C.Y.; Shih, C.; Chiang, T.; Chang, E.; Lee, Y.F.; Tsai, M.Y.; Chang, C.; Lee, K.H. J. Med. Chem. 2002, 45, 5037.

16. Jha, A.; Zhao, J.; Cameron, T. S.; DeClercq, E.; Manavathu, E. K.; Stables, J. P. Lett. Drug Design Discov. 2006, 3, 304.

17. Ohtsu, H.; Itokawa, H.; Xiao, Z.; Su, C.Y.; Shih, C.C.Y.; Chiang, T.; Chang, E.; Lee, Y.; Chiu, S.Y.; Chang, C.; Lee, K.H. Bioorg. Med. Chem. 2003, 11, 5083.

18. Babu, K. V. D.; Rajasekharan, K. N. Org. Prep. Proc. Int'l. 1994, 26, 674.

19. Carruthers, W. In Some Modern Methods of Organic Synthesis $3^{\text {rd }}$ Edition, Cambridge University Press: Cambridge (UK) 1998, p 8.

20. Samula, K.; Kardasz, K. Pol. J. Chem. 1985, 59, 73.

21. Gu, J.-X.; Li, Z.-Y.; Lin, G.-Q. Tetrahedron Asymm. 1992, 3, 1523.

22. Azarifar, D.; Ghasemnejad, H. Molecules, 2003, 8, 642.

23. Esmaeili, A. A.; Tabas, M. S.; Nasseri, M. A.; Kazemi, F. Monatsh. Chem., 2005, 136, 571.

24. Lidström, P.; Tierney, J.; Wathey, B.; Westman, J. Tetrahedron 2001, 57, 9225.

25. El Ashry, E. S. H.; Kassem, A. A. Arkivoc 2006, (ix), 1. 